\title{
La teoría de la virtud argumentativa: ¿un mero complemento moral?
}

\section{Virtue argumentation theory: a mere moral complement?}

\section{José Ángel Gascón}

Centro de Investigación en Educación y Desarrollo (CIEDE)

Universidad Católica de la Santísima Concepción (UCSC)

Alonso de Ribera 2850, Concepción, Chile

igascon@ucsc.cl

Artículo recibido: 22-03-2018

Artículo aceptado: 02-12-2018

\section{RESUMEN}

El lugar que le corresponde a la teoría de la virtud argumentativa en el campo de los estudios de la argumentación ha sido recientemente comentado por Gensollen, quien propone caracterizarla como una teoría complementaria que se ocupa de la evaluación moral. Frente a esta valoración, en el presente artículo sostengo que una perspectiva de la virtud en argumentación no se limita a la evaluación moral, sino que también es pertinente para el estudio de la cognición y el razonamiento humanos. Además, critico tal distinción entre teorías "complementarias" y "fundamentales", así como el criterio que Gensollen usa para demarcarlas.

PALABRAS CLAVE: moral, pragmadialéctica, propósito de las teorías, razonamiento, sesgos cognitivos, virtud argumentativa

\begin{abstract}
The place that belongs to virtue argumentation theory in the field of argumentation studies has been recently discussed by Gensollen, who proposes that it should be characterized as a complementary theory that deals with moral evaluation. Against this assessment, in the present article I argue that a virtue approach to argumentation is not restricted to moral evaluation, but it is also relevant to the study of human cognition and reasoning. Moreover, I criticize such a distinction between "complementary" and "fundamental" theories, as well as the criterion Gensollen uses in order to demarcate them.
\end{abstract}

KEYWORDS: argumentative virtue, cognitive bias, morals, purpose of theories, pragma-dialectics, reasoning 


\section{INTRODUCCIÓN}

Cuando una nueva teoría surge en alguna disciplina filosófica, habitualmente el propósito del teórico es uno de los dos siguientes:

a) Explicar mejor un fenómeno que las teorías existentes no explican satisfactoriamente.

b) Explicar un fenómeno del que las teorías existentes no se ocupan.

Existe, por supuesto, la posibilidad de que alguna teoría sea planteada con ambos propósitos, de modo que la pretensión no es solo que la teoría es más exacta sino también más general. La pregunta que me interesa aquí es: ¿cómo debemos evaluar en cada caso el éxito explicativo de la teoría? Si se trata de una teoría con el propósito (a), parece claro que su éxito dependerá de si dicha teoría es capaz de explicar el fenómeno en cuestión de manera más satisfactoria que las demás. En el caso de (b), la novedad de la perspectiva teórica exige, en primer lugar, que se muestre el interés filosófico del fenómeno, y, en segundo lugar, que se lo pueda explicar cumpliendo con unos estándares mínimos de rigor filosófico.

A partir de esta distinción, es fácil ver que una teoría cuyo propósito es (b) puede ser mal comprendida si se la confunde con una teoría cuyo propósito sea (a), o incluso si se pretende que cumpla con (a) y (b). Los partidarios de teorías con una perspectiva más tradicional, que se ocupan de un fenómeno conocido y largamente estudiado, son particularmente susceptibles de cometer este error. Sin embargo, es posible que el teórico que se centra en (b) considere que ninguna teoría puede cumplir con (a) y (b) al mismo tiempo, de modo que su modesto objetivo sea simplemente limitarse a explicar el nuevo fenómeno. Existe, puede pensar el teórico, un fenómeno importante que no ha sido tenido suficientemente en cuenta. Las perspectivas tradicionales no nos permitían verlo. Se hace, pues, necesario adoptar una perspectiva que, aunque deje a un lado otros fenómenos, nos permita lidiar con este que nos ocupa.

Estas reflexiones vienen al caso de la aparición relativamente reciente de una perspectiva de la virtud en argumentación. En un ambiente filosófico preocupado principalmente por las propiedades lógicas, dialécticas o retóricas de los mensajes argumentativos de los hablantes, Daniel Cohen (2005) llamó la atención sobre ciertos fenómenos que tenían más que ver con características del argumentador. En su comentario al artículo de Cohen, Andrew Aberdein señaló que aquello podía ser el 
origen de una teoría de la virtud argumentativa, centrada en los rasgos del carácter de los argumentadores en lugar de los argumentos como productos. El resultado, con el tiempo, fue un artículo de Aberdein (2010) en el que ponía explícitamente sobre la mesa la propuesta de desarrollar tal teoría y bosquejaba el aspecto que podría tener el camino que iba a hacerse al andar en esa dirección.

¿Ante qué tipo de teoría nos encontramos? ¿Cuál es su propósito? En un artículo publicado en esta misma revista, Mario Gensollen comenta algunas de las fortalezas y debilidades de una perspectiva de la virtud en argumentación y concluye que esta teoría "solo puede ser un enfoque complementario al enfoque lógico y al pragma-dialéctico" (Gensollen, 2017: 56). El presente artículo es una respuesta a esa valoración de Gensollen. Considero que su postura es representativa del recibimiento que dan a las teorías novedosas algunos teóricos que adoptan un enfoque tradicional -al menos, aquellos que llegan a reconocer el valor de tal novedad-. Para quien tiene un martillo, el foco de interés se sitúa alrededor de los clavos, y los demás objetos-destornilladores, pinzas, metro- poseen un interés tangencial solo en la medida en que permitan lidiar con los clavos. De forma similar, desde un punto de vista lógico o dialéctico, puede ocurrir que se parta de la expectativa de que una nueva teoría debe ocuparse -al menos- del discurso argumentativo, de los argumentos. De este modo, se comete, creo yo, el error de exigir a esa nueva teoría que cumpla con el propósito (a), o incluso una combinación de (a) y (b). En caso contrario, si se limita a (b), entonces -se cree- no puede ser más que un mero complemento de las teorías que se ocupan del meollo de la cuestión.

Gensollen hace varias reflexiones que sustentan su juicio sobre el lugar que le corresponde a una perspectiva de la virtud en la empresa común dedicada al estudio de nuestras prácticas argumentativas. En las siguientes secciones trataré cada una de esas observaciones por separado: en la sección 2, discutiré la consideración de que las cualidades de los argumentadores solo son pertinentes desde un punto de vista moral; $y$, en la sección 3, comentaré el uso que hace Gensollen de los componentes del programa de investigación de la pragmadialéctica para distinguir entre un enfoque "fundamental" y uno "complementario".

\section{LA TEORÍA DE LA VIRTUD ARGUMENTATIVA COMO PERSPECTIVA MORAL}

Los partidarios de una perspectiva de la virtud en argumentación han enfatizado -es cierto- la relevancia de algunas cuestiones morales y la importancia de mantener una 
actitud cooperativa en nuestros intercambios argumentativos. Ya Cohen (1995) -antes incluso de izar la bandera de la teoría de la virtud argumentativa-criticó la concepción que la filosofía tenía de la argumentación como un combate en el que hay ganadores y perdedores. Yo mismo (Gascón, 2015) propuse que, en lo que se refiere al estudio del argumento como producto, una teoría de la virtud argumentativa podría arrojar algo de luz sobre aspectos que conciernen a la moral -entre otros-. A partir de estas referencias -y de otras menos afortunadas ${ }^{1}-$ Gensollen infiere que el carácter de los argumentadores solo es pertinente para la evaluación moral y el fomento de una actitud cooperativa. Entre sus conclusiones nos encontramos con la siguiente afirmación:

No obstante, la consideración del agente está restringida solo a la evaluación moral de las argumentaciones y no a otros tipos de normatividad que son susceptibles de una sistematización mayor a partir de otros elementos de nuestras prácticas. (Gensollen, 2017: 56-57)

A pesar de este limitado papel que Gensollen atribuye a una perspectiva de la virtud, él asegura que las virtudes argumentativas son tan imprescindibles como las habilidades lógicas y dialécticas, ya que "los vicios argumentativos muchas veces pueden malograr los objetivos cooperativos que buscamos alcanzar cuando argumentamos" (Ibid. 53). En resumidas cuentas, el valor de las virtudes argumentativas parece reducirse a que facilitan la cooperación.

Para ver que esto es un error, conviene enfatizar que la teoría de la virtud argumentativa se presenta con un carácter eminentemente práctico -como el propio Gensollen reconoce (Ibid.)-. El hecho de que las discusiones argumentativas tengan lugar invariablemente entre personas no solo tiene repercusiones morales. También es pertinente para la evaluación y mejora de nuestras capacidades cognitivas y racionales. No somos máquinas que produzcan unos resultados a partir de un algoritmo, de tal modo que bastase con revisar las líneas de código para evaluar y mejorar nuestro razonamiento -tarea que podría encomendarse en exclusiva a la lógica-. Nuestro conocimiento y nuestras decisiones dependen de muchos factores, algunos de los cuales tienen que ver con nuestro carácter.

Esto es precisamente lo que sostiene Correia (2012) al proponer una ética² de

\footnotetext{
${ }^{1}$ Gensollen (2007: 52) cita a Schreier, Groeben y Christmann (1995), quienes proponen unos criterios de evaluación ética de las discusiones argumentativas, como partidarios de una perspectiva de la virtud en argumentación; pero estos autores son psicólogos que abordan el estudio de la argumentación desde un enfoque empírico y nada tienen que ver con la teoría de la virtud. Asimismo, en esa misma página, aparece citado Correia (2012), quien no se apoya en la evaluación moral sino en los sesgos cognitivos -de los que hablaré a continuación- para defender una perspectiva de la virtud en argumentación.

${ }^{2}$ Correia no usa "ética" para referirse a la reflexión moral sino a todo lo relacionado con el carácter de la persona en general.
} 
la argumentación. Correia se apoya en la existencia -hoy ya suficientemente acreditada- de numerosos sesgos cognitivos que nos conducen sistemáticamente hacia errores de razonamiento. Se sabe, por ejemplo, que los seres humanos tendemos a evaluar la solidez de los argumentos en función de si estamos de acuerdo con su conclusión (Correia, 2012: 228), a interpretar la información de manera que confirme nuestras creencias previas (Ibid. 228-229) y a sobrestimar nuestras cualidades positivas al mismo tiempo que subestimamos las negativas, lo que incluye la tendencia a subestimar nuestros propios sesgos (Ibid. 230). El punto central que defiende Correia es que, dado que estas ilusiones cognitivas se producen de manera inconsciente, no se trata de errores que puedan solucionarse animando a los argumentadores a respetar las reglas de la lógica y la dialéctica. Los esfuerzos intencionales no son suficientes para acabar con los sesgos.

Uno de los elementos que influye en nuestra manera de razonar y que acostumbra a causar la aparición de sesgos es precisamente algo que, tanto en ética como en epistemología de la virtud, es considerado por muchos como un componente definitorio de las virtudes: la motivación. La psicóloga social Ziva Kunda (1990) mostró, sobre la base de numerosos estudios, que las motivaciones afectan a nuestros razonamientos. Kunda distinguió dos tipos de motivaciones: (1) la motivación de llegar a una conclusión correcta, sea cual sea, y (2) la motivación de llegar a una conclusión determinada. Mientras que el primer tipo de motivación nos mueve a invertir un mayor esfuerzo cognitivo y a razonar de manera más objetiva, el segundo tipo propicia la aparición de sesgos.

Estas motivaciones del segundo tipo influyen en la selección de premisas y de reglas inferenciales (Kunda 1990: 489). De este modo, los sesgos no se manifiestan necesariamente bajo la forma de errores en las creencias o en las inferencias, sino en la selección previa de las creencias y las reglas inferenciales que se usarán en el razonamiento. Tras esa selección sesgada, es posible que el razonamiento mismo sea correcto. Este hecho sugiere que el análisis -desde una perspectiva lógica- del discurso argumentativo resultante no basta para detectar las malas artes de los sesgos. Se hace imprescindible lidiar con las motivaciones previas al razonamiento.

Por esta razón, Correia nos urge a tomar en consideración el carácter de los argumentadores, ya que de lo contrario nuestras teorías de la argumentación no serán más que castillos en el aire que se asemejan poco a lo que sucede en el mundo real (2012: 226-227): 
En otras palabras, mi sugerencia es que una teoría normativa de la argumentación solo puede ser una herramienta eficaz con consecuencias prácticas, en lugar de un mero ideal sobre cómo debería argumentar la gente en el mejor de los mundos, si tiene en cuenta lo que indican los estudios empíricos sobre la manera en que la gente suele razonar realmente en los debates cotidianos.

De hecho, durante los últimos años, los educadores y los investigadores en pensamiento crítico han enfocado su atención en el problema de los sesgos cognitivos, más que en el análisis de estructuras argumentativas. Es más, Thagard (2011) sostiene que las habilidades para formar creencias y tomar decisiones no mejoran por medio del estudio de la lógica -formal o informal-. En lugar de ello, el pensamiento crítico debe tomar nota de los descubrimientos en la investigación psicológica sobre los errores de razonamiento a los que somos propensos. Sobre ello, Thagard afirma:

Sin embargo, existen abundantes pruebas de la psicología cognitiva y de la neurociencia de que las inferencias humanas son realmente paralelas en lugar de seriales, multimodales en lugar de simplemente basadas en el lenguaje y tan emocionales como cognitivas. (Thagard 2011: 154)

En efecto, en el razonamiento humano, las emociones juegan un papel tan fundamental como las capacidades cognitivas. Las emociones no son simples distracciones o intrusos ajenos al razonamiento y la cognición, sino que resultan ser una "parte integral de la forma como el cerebro controla el flujo de información" (Ibid. 155). Esto puede explicar que se produzcan sesgos basados en inferencias motivadas -como nos acaba de mostrar Kunda más arriba-. Thagard asegura que "sería inútil intentar capturar estas inferencias como argumentos obviamente falaces" (Ibid. 157), pues no se trata de inferencias seriales que se produzcan de manera consciente, sino que son paralelas e inconscientes. Él presenta ejemplos tales como la negación del cambio climático antropogénico -causada por motivaciones políticas- o de la teoría de la evolución causada por motivaciones religiosas-. En tales casos, concluye, "el pensamiento crítico requiere una comprensión psicológica de la inferencia motivada más que una comprensión lógica de la estructura del argumento" (Ibid. 158).

No se me escapa el hecho de que, al hablar de Thagard, nos hemos deslizado subrepticiamente hacia el pensamiento crítico y las inferencias, cuando el tema principal de este artículo es la argumentación. Sin embargo, creo que es muy pertinente por una razón fundamental. Recordemos que he enfatizado el aspecto práctico de una teoría de la virtud argumentativa, así que nos interesa la utilidad de la teoría para formar buenos argumentadores. Y, aun si distinguimos -como creo que deberíamos- entre las inferencias como un fenómeno psicológico y los argumentos como un fenómeno público, debemos abandonar toda esperanza de mejorar estos si no nos ocupamos antes de 
aquellas.

Así, vemos que las motivaciones y las emociones, fenómenos que dependen del carácter y a los que una perspectiva de la virtud en argumentación presta especial atención, son fundamentales para comprender nuestras creencias y decisiones. El carácter de los argumentadores no solo es pertinente para la evaluación moral. Al contrario, nuestra forma de razonar solo puede estudiarse seriamente y con expectativas realistas de mejora si se toman en consideración esos rasgos que nos hacen humanos.

\section{EL CARÁCTER COMPLEMENTARIO DE LA TEORÍA DE LA VIRTUD ARGUMENTATIVA}

Como espero haber mostrado, una perspectiva de la virtud en argumentación no es una teoría situada al margen que solo se ocupe de los aspectos morales de las discusiones críticas. No es ajena a algunos de los principales propósitos de la lógica y de la dialéctica: formar creencias plausibles, tomar buenas decisiones, argumentar de manera razonable. Pero existen, evidentemente, elementos de la argumentación que una teoría de la virtud argumentativa no puede estudiar apropiadamente, tales como los vínculos lógicos entre proposiciones y el uso que los hablantes hacen del lenguaje. Volvamos ahora por un momento la vista atrás hacia las reflexiones planteadas en la Introducción sobre los propósitos de las teorías y los malentendidos que pueden darse acerca de los mismos. Recordemos que una teoría puede presentarse con alguno de dos propósitos:

a) Explicar mejor un fenómeno que las teorías existentes no explican satisfactoriamente.

b) Explicar un fenómeno del que las teorías existentes no se ocupan.

Cuestiones tales como las conexiones lógicas pertenecen a lo que podría llamarse un fenómeno conocido. Por otro lado, el papel del carácter de los individuos en la argumentación puede considerarse un fenómeno de reciente constatación precisamente el que una teoría de la virtud argumentativa se propone tratar-. En otra parte (Gascón, 2015: 482) he defendido que no debemos tratar cuestiones como la calidad de los argumentos desde una perspectiva de la virtud. Esto implica que, de las dos posibilidades planteadas, el propósito de una teoría de la virtud argumentativa se limita a (b).

¿En qué lugar nos deja eso? Para Gensollen, la renuncia al propósito (a) hace 
que dicha teoría deba ser relegada a un papel "complementario", mientras que las teorías que cumplen tal propósito pueden ser consideradas "fundamentales". Más concretamente, se sirve del siguiente criterio para distinguir entre unas y otras:

Lo primero que debemos dejar asentado es que cualquier enfoque en Teoría de la argumentación debe estar compuesto de distintos elementos: uno filosófico (una concepción de la razonabilidad), uno teórico (un modelo de la argumentación), uno analítico (una reconstrucción sistemática del discurso argumentativo), uno práctico (la mejora de las prácticas y habilidades argumentativas), y uno empírico (factores y procesos que determinan la realidad argumentativa). (Gensollen, 2017: 53)

El lector ya se habrá percatado de que estos elementos son los cinco constituyentes que, según los proponentes de la teoría pragmadialéctica, son componentes "necesarios" de "un programa completo de investigación" en argumentación (Eemeren y Grootendorst, 2004: 11). En particular, estos cinco componentes caracterizan al programa de investigación que los pragmadialécticos denominan pragmática normativa.

A este respecto, debo señalar antes que nada que tengo serias dudas de que la escuela pragmadialéctica conciba tales componentes de una manera tan fuerte, como una condición necesaria para poder considerar que una teoría es "fundamental". Lo cierto es que esos autores se refieren a ellos como componentes de un programa de investigación, no de una teoría aislada ${ }^{3}$. La función de la teoría que motiva su correspondiente programa de investigación es proporcionar un determinado enfoque de la argumentación como punto de partida. Los cinco componentes, en cuanto que diferentes tareas que se llevan a cabo en un programa de investigación, difícilmente pueden concebirse como criterios de demarcación de teorías.

En cualquier caso, sea cual sea la opinión de los pragmadialécticos, es evidente que para Gensollen eso funciona como criterio (Gensollen 2017, 53, n. 9). De acuerdo con tal criterio, pues, él resuelve que la teoría de la virtud argumentativa carece de los componentes filosófico, teórico y analítico, y por lo tanto solo puede desempeñar el papel de complemento de otros enfoques. Lamentablemente, el veredicto se nos presenta sin unas razones que lo sustenten. A pesar de ello, por fortuna, al menos si nos limitamos al componente analítico, contamos con una declaración de culpabilidad. Como he señalado más arriba, creo que una teoría de la virtud argumentativa no debe ocuparse del análisis y evaluación del discurso argumentativo. ¿Caso cerrado, entonces?

Las cosas no son tan sencillas. Creo que existe aquí un malentendido que puede

${ }^{3}$ Véase, por ejemplo, Eemeren (1990), Eemeren y Grootendorst (2004: 11), y Eemeren et al. (2014: 520). 
entenderse sobre la base de mi distinción entre (a) teorías que explican fenómenos conocidos y (b) teorías que llaman la atención sobre nuevos fenómenos. Para ver lo que ha ocurrido, comencemos por situar el discurso argumentativo entre los fenómenos que pertenecen a (a), y el carácter del argumentador, incluyendo sus emociones y sus motivaciones, entre los fenómenos que pertenecen a (b) -una clasificación que no debería suscitar controversia-. Mis objeciones al veredicto de Gensollen son, en primer lugar, que se está exigiendo ilegítimamente a la teoría de la virtud argumentativa que sea tanto (a) como (b); y, en segundo lugar, que ha asumido con demasiada celeridad que la pragmadialéctica -o, para el caso, la lógica- no necesita ocuparse de (b).

Las dos objeciones están relacionadas. Ambas comparten una crítica a la idea que subyace en la valoración de Gensollen- de que los aspectos de la argumentación que pertenecen a (a) constituyen el meollo o lo fundamental de la argumentación, mientras que los aspectos que pertenecen a (b) son secundarios. Para mostrar que esto no está tan claro, argumentaré que el hecho de dejar a un lado el carácter de los argumentadores dificulta mucho -si no imposibilita- la tarea de elaborar una concepción satisfactoria de la razonabilidad -el componente filosófico-. En consecuencia, si una teoría no tiene en cuenta el fenómeno que pertenece a (b), no le será fácil cumplir con el criterio, propuesto por el propio Gensollen, que identifica a una teoría "fundamental".

Veamos qué concepción de la razonabilidad propone la pragmadialéctica. Eemeren y Grootendorst (2004: 16) defienden una perspectiva "crítica" de la razonabilidad $^{4}$, que se basa en el presupuesto de que nunca podemos estar completamente seguros de nada y que promueve la discusión argumentativa para que nuestros puntos de vista sean sometidos sistemáticamente a la crítica de otros. La argumentación se ve, desde esta perspectiva, como un medio de resolver una diferencia de opinión de acuerdo con unas reglas que deben ser aceptables para los participantes y adecuadas para que la discusión crítica sea razonable. Así, la norma de la razonabilidad que propone la pragmadialéctica cumple dos criterios (Ibid., 17): la validez del problema -las reglas deben servir para resolver adecuadamente la diferencia de opinión- y la validez intersubjetiva -las reglas deben ser aceptables para todas las partes en una discusión-.

Aunque no es lo que más me interesa aquí, vale la pena dejar constancia de que Popa (2016) ha mostrado convincentemente que el primer criterio es circular y por tanto carece de utilidad para evaluar la validez de las reglas pragmadialécticas. Las reglas,

${ }^{4}$ Frente a las perspectivas "geométrica" y "antropológica". Los autores toman esta distinción entre tres visiones de la razonabilidad de Toulmin (1976). 
sostienen los pragmadialécticos, cumplen el criterio de la validez del problema si sirven para mantener una discusión dentro del ámbito de lo razonable y para evitar falacias. ¿Cómo podemos comprobar si ese es el caso? En primer lugar, debemos averiguar qué se entiende por "razonable" y por "falacia". Pero inmediatamente caemos en la cuenta de que las definiciones de "razonable" y de "falacia", en la pragmadialéctica, se apoyan en las reglas mismas: las falacias se definen como violaciones de alguna de tales reglas. Como señala Popa, "el carácter "problemático" de las situaciones en las que se violan las reglas parece consistir en el hecho de que las reglas han sido violadas' (Popa, 2016: 197), de tal modo que "las reglas han llegado para solucionar problemas que no podrían no solucionar" (Ibid. 198). Así, vemos que en efecto la pragmadialéctica cumple el criterio de validez del problema, pero de una manera absolutamente trivial.

Esa cuestión atañe a la coherencia de la concepción pragmadialéctica de la razonabilidad, es decir, a la cuestión de si tal concepción cumple con los criterios que la pragmadialéctica misma propone. Sin embargo, lo que más me interesa comentar para mis propósitos es su suficiencia, es decir, la cuestión de si la pragmadialéctica proporciona una explicación teórica completa de la razonabilidad. ¿Da cuenta de la razonabilidad de manera satisfactoria la perspectiva pragmadialéctica?

Me parece muy difícil responder afirmativamente a esa pregunta. La razonabilidad, incluso si nos limitamos al contexto de la argumentación, es un concepto mucho más rico que la simple idea de que debemos estar abiertos a la posibilidad de estar equivocados y a la discusión crítica. Con esto no pretendo sostener simplemente que tal concepción puede mejorarse -algo que puede decirse de prácticamente cualquier idea filosófica-; lo que sostengo es que es manifiestamente insatisfactoria. Las reglas pragmadialécticas establecen, por ejemplo, que, si un punto de vista ha sido concluyentemente justificado o refutado, entonces se considera justificado o refutado únicamente dentro de los estrictos límites de la discusión crítica correspondiente. Como los propios autores admiten (van Eemeren y Grootendorst, 2004: 136), esto tiene la consecuencia de que una persona puede iniciar otra discusión en la que defienda el mismo punto de vista que acaba de ser refutado -tantas veces como le apetezca, como una recreación del día de la marmota en la famosa película Atrapado en el tiempo (1993)-. Me atrevo a asegurar que ninguno de nosotros consideraríamos que tal persona se comporta de manera razonable.

Además de eso, el principio de externalización de la pragmadialéctica le impide prestar atención al carácter y los estados psicológicos de los argumentadores; las reglas se aplican solo a sus proferencias. Como ya argumenté en otra parte (Gascón, 2017), 
ese principio es muy apropiado para una teoría que se ocupa del análisis y la evaluación del discurso argumentativo, pero incapacita para explicar todo lo que involucra la compleja y rica noción de argumentar razonablemente. Una de las principales razones de esto ya se vio en la sección anterior. No es posible tratar la cuestión de la argumentación razonable sin tomar en consideración la psicología del razonamiento y los sesgos cognitivos, y para ello debemos fijarnos -entre otras cosas- en las motivaciones y las emociones humanas. Como señala Thagard -aunque él hable de "racionalidad" en lugar de "razonabilidad"-, el asunto no se limita a la calidad de los argumentos:

Desde esta perspectiva, la racionalidad no es simplemente una cuestión de usar buenos argumentos y evitar argumentos falaces, sino más bien una cuestión de adoptar patrones de pensamiento y de comportamiento que satisfagan mejor metas legítimas sobre qué creer y qué hacer. (Thagard 2011: 156)

En conclusión, parece que, según el criterio de Gensollen, la pragmadialéctica tampoco puede considerarse una teoría "fundamental". La moraleja que debemos sacar de todo esto no es que una teoría de la virtud argumentativa sea más "fundamental" o proporcione una mejor concepción de la razonabilidad. Creo, más bien, que carece de sentido hablar de teorías "fundamentales" y teorías "complementarias". La perspectiva de la virtud en argumentación ha llamado la atención sobre unos fenómenos que la lógica y la dialéctica habían desatendido. Estos fenómenos, desde esa perspectiva, no son secundarios sino que poseen una importancia similar a los fenómenos que hasta ahora habían acaparado equivocadamente la atención de los teóricos. Tal cambio de enfoque, que dirige nuestra mirada a nuevos aspectos de la argumentación, es precisamente uno de los méritos de la perspectiva de la virtud.

En este sentido, puede trazarse un paralelismo con lo que ha sucedido con las teorías de la virtud en epistemología. Sin duda, algunas teorías en epistemología de la virtud han continuado con la acostumbrada tarea de proporcionar una definición del conocimiento basada en condiciones necesarias y suficientes (cf. Sosa, 1991; Zagzebski, 1996). Pero otros teóricos, en cambio, han reparado en las posibilidades que ofrece el nuevo enfoque para arrojar un poco de luz sobre aspectos de nuestras prácticas epistémicas que habían sido descuidados por los enfoques tradicionales (cf. Code, 1987; Roberts y Wood, 2007). Estos teóricos han renunciado explícitamente a llevar a cabo la tarea definitoria y se han centrado en estudiar cuestiones más relacionadas con, por ejemplo, la actividad cognitiva, las comunidades epistémicas y nuestra actitud epistémica ante el mundo. Como señala Hookway (2003), la 
epistemología de la virtud ofrece una perspectiva en la que los conceptos de "conocimiento" y de "justificación" resultan ser menos centrales. Un cambio similar de perspectiva es que el ofrece, en mi opinión, la teoría de la virtud argumentativa.

Por todo ello, parece fuera de lugar pedir a una teoría que propone un nuevo enfoque que se ocupe de los mismos asuntos de los que se ocupaban los enfoques tradicionales. Exigir a una teoría de la virtud argumentativa que ofrezca un método de análisis del discurso argumentativo me resulta tan desencaminado como lo sería exigir a la lógica que dé cuenta del papel que desempeñan las motivaciones en nuestro razonamiento. Cada teoría se ocupa de su objeto de estudio. Cada una esclarece una modesta parte de ese conjunto de fenómenos al que nos referimos como la categoría genérica de "argumentación". Ninguna proporciona una imagen completa de nuestras prácticas argumentativas.

\section{CONCLUSIÓN}

Uno de los principales puntos de interés de una perspectiva de la virtud en argumentación es que aleja el foco de atención de los elementos que han sido tradicionalmente considerados centrales -los argumentos como productos y sus conexiones lógicas- y lo acerca a aspectos que hasta ahora han sido pasados por alto -las motivaciones, la actitud, las disposiciones de los argumentadores-. Por esta razón, para aquellos que mantengan una perspectiva tradicional de la argumentación, una teoría de la virtud argumentativa parece tener un interés limitado o ser meramente accesoria, pues no se enfoca en lo que, desde su punto de vista, es lo importante.

Este es el error que, según he argumentado, comete Gensollen al considerar que la teoría de la virtud argumentativa es meramente "complementaria" porque no permite analizar aspectos tales como el discurso argumentativo. Mi respuesta ha consistido en subrayar el hecho de que el objetivo de esta nueva perspectiva es centrarse en fenómenos hasta ahora considerablemente desatendidos, no ocuparse de aquello que ya tratan otras teorías. Asimismo, he argumentado que este nuevo campo de estudio -el carácter de los argumentadores- no es de ningún modo un aspecto secundario o tangencial de la argumentación; es un elemento imprescindible para comprender cómo razonamos y cómo nos comportamos los seres humanos durante una discusión argumentativa.

Creo, en fin, que no tiene mucho sentido hablar sobre qué teorías son "fundamentales" y cuáles son "complementarias", pues tal debate contribuye poco a 
nuestra comprensión de la práctica argumentativa. No obstante, si se emprende este debate, los criterios deben ser coherentes. Cuando una teoría propone una nueva perspectiva que arroja luz sobre nuevos fenómenos, solo se le puede exigir a dicha teoría que dé cuenta de los fenómenos tradicionales si, al mismo tiempo, también se exige a las teorías tradicionales que den cuenta de los fenómenos nuevos. Si el hecho de que una teoría de la virtud argumentativa no sirva para analizar la estructura de los argumentos se considera como una insuficiencia, entonces también se debe considerar como una insuficiencia el hecho de que la lógica y la dialéctica no sirvan para explicar y reducir significativamente los sesgos cognitivos. Por ello, ante quien insista en mantener las categorías de teorías "fundamentales" y teorías "complementarias", mi respuesta solo puede ser una: todas las teorías son complementarias.

\section{REFERENCIAS}

Aberdein, A. (2010). "Virtue in argument". Argumentation, 24(2), 165-179.

Code, L. (1987). Epistemic responsibility. Hanover: University Press of New England.

Cohen, D. H. (1995). "Argument is war... and war is hell: Philosophy, education, and metaphors for argumentation". Informal Logic, 17(2), 177-188.

Cohen, D. H. (2005). "Arguments that backfire". En: D. Hitchcock y D. Farr (Eds.), The uses of argument: Proceedings of the sixth conference of the Ontario Society for the Study of Argumentation (pp. 58-65). Hamilton, ON: OSSA.

Correia, V. (2012). "The ethics of argumentation". Informal Logic, 32(2), 222-241. - (2017). "Accountability breeds response-ability: Contextual debiasing and accountability in argumentation". En: P. Brézillon, R. Turner, y C. Penco (Eds.), Modeling and using context. 10th international and interdisciplinary conference, CONTEXT 2017 Paris, France, June 2023, 2017 (pp. 127-136). Cham: Springer.

Eemeren, F. H. van. (1990). "The study of argumentation as normative pragmatics". Text, 10(1/2), $37-44$.

Eemeren, F. H. van, Garssen, B., Krabbe, E. C. W., Snoeck Henkemans, A. F., Verheij, B., y Wagemans, J. H. M. (2014). Handbook of argumentation theory. Dordrecht: Springer.

Eemeren, F. H. van, y Grootendorst, R. (2004). A systematic theory of argumentation. Nueva York: Cambridge University Press.

Gascón, J. Á. (2015). "Arguing as a virtuous arguer would argue". Informal Logic, 35(4), 467-487. - (2017). "Brothers in arms: Virtue and pragma-dialectics". Argumentation, 31(4), 705-724.

Gensollen, M. (2017). "El lugar de la teoría de la virtud argumentativa en la teoría de la argumentación contemporánea". Revista Iberoamericana de Argumentación, 15, 41-59.

Hookway, C. (2003). "How to be a virtue epistemologist". En: M. DePaul y L. Zagzebski (Eds.), Intellectual virtue: Perspectives from ethics and epistemology (pp. 183-202). Nueva York: Oxford University Press.

Kunda, Z. (1990). "The case for motivated reasoning". Psychological Bulletin, 108(3), 480-498.

Popa, E. O. (2016). "Criticism without fundamental principles". Informal Logic, 36(2), 192-216.

Roberts, R. C., y Wood, W. J. (2007). Intellectual virtues: An essay in regulative epistemology. Nueva York: Oxford University Press.

Schreier, M., Groeben, N., y Christmann, U. (1995). "That's not fair! Argumentational integrity as an ethics of argumentative communication". Argumentation, 9(2), 267-289.

Sosa, E. (1991). Knowledge in perspective: Selected essays in epistemology. Cambridge: Cambridge University Press. 
Thagard, P. (2011). "Critical thinking and informal logic: Neuropsychological perspectives". Informal Logic, 31(3), 152-170.

Toulmin, S. (1976). Knowing and acting. Nueva York: Macmillan.

Zagzebski, L. T. (1996). Virtues of the mind. New York: Cambridge University Press.

AGRADECIMIENTOS: Debo dar las gracias en primer lugar a Mario Gensollen por su interés en la teoría de la virtud argumentativa y por darme la oportunidad de discutir el lugar que le corresponde a dicha teoría en los estudios de argumentación. A Paula Olmos también le estoy agradecido por animarme a elaborar una respuesta al artículo de Gensollen. Este trabajo ha sido posible gracias al proyecto "La construcción de agentes argumentativos en las prácticas del discurso público" (FFI2014-53164-P).

JOSÉ ÁNGEL GASCÓN: Doctor en filosofía por la UNED (Madrid) y actualmente becario postdoctoral en la UCSC (Concepción, Chile). Su tesis doctoral consistió en la propuesta de una perspectiva de la virtud en argumentación. Varios de los capítulos de su tesis han sido publicados en Topoi, Informal Logic, Argumentation y en esta misma Revista Iberoamericana de Argumentación. 